\title{
An approach to the uniform Artin-Rees the- orems from the notion of relation type
}

FRANCESC PLANAS-VILANOVA, Departament de Matemàtica Aplicada 1, Universitat Politècnica de Catalunya, Barcelona, Spain

E-mail: francesc.planas@upc.es

\section{Introduction}

Our purpose in this work is to emphasize the tight connection between the Artin-Rees lemma and the notion of the relation type of an ideal with respect to a module. We present new proofs of some already known uniform ArtinRees theorems as well as a new approach to the subject.

We begin by recalling the well-known Artin-Rees lemma, which plays an essential role in the completion process of rings and modules (see [2], [3] [17], [18], [29], [40] for proofs, consequences and historical notes). The standard proof will provide us with perhaps the first evidence of the above cited strong connection between the Artin-Rees lemma and the notion of relation type, since it involves what is nowadays called the Rees module of an ideal with respect to a module.

Lemma 1.1 The Artin-Rees Lemma Let $A$ be a noetherian ring, $I$ an ideal of $A$ and let $N \subseteq M$ be two finitely generated $A$-modules. Then there exists an integer $s \geq 1$, depending on $N, M$ and $I$, such that for all $n \geq s$,

$$
I^{n} M \cap N=I^{n-s}\left(I^{s} M \cap N\right) .
$$

Proof: Let $\mathcal{R}(I)=\bigoplus_{n>0} I^{n}$ be the Rees ring of the ideal $I$, which is noetherian since $A$ is noetherian. Let $\mathcal{R}(I ; M)=\bigoplus_{n \geq 0} I^{n} M$ be the Rees module of $I$ with respect to the module $M$, which is a graded finitely generated $\mathcal{R}(I)$-module since $M$ is finitely generated. Consider the graded submodule $L=\bigoplus_{n>0} I^{n} M \cap N$, which is finitely generated since $\mathcal{R}(I ; M)$ is a noetherian module. Take $s$ as the largest degree of a set of generators. Then $L_{n} \subset I^{n-s} L_{s}$ for all $n \geq s$, which implies the assertion. 
If we denote by $E(N, M ;\{I\})_{n}=I^{n} M \cap N / I\left(I^{n-1} M \cap N\right)$, then the lemma is equivalent to stating that there exists an integer $s \geq 1$, depending on $N, M$ and $I$, such that $E(N, M ;\{I\})_{n}=0$ for all $n \geq s+1$. A weaker result, which follows immediately from the lemma, and which will play an important role on the sequel, is that there exists an integer $s \geq 1$, depending on $N, M$ and $I$, such that $I^{n} M \cap N \subseteq I^{n-s} N$.

\section{A problem posed by Eisenbud and Hoschster}

Eisenbud and Hochster in [7], originally motivated by a question of Wehrfritz about the existence of a uniform index of nilpotency (Corollary 2 in [7]), gave a new proof of Zariski's main lemma on holomorphic functions on an algebraic variety ([43]) by establishing what they called a "Nullstellensatz with nilpotents". They also remarked that a different approach to their Theorem could be obtained by answering the following question of uniform Artin-Rees type:

QUESTION 2.1 Let $A$ be an affine ring and let $N \subseteq M$ be two finitely generated $A$-modules. Does there exist an integer $s \geq 1$ such that, for all integers $n \geq s$ and for all maximal ideals $\mathfrak{m}$ of $A$,

$$
\mathfrak{m}^{n} M \cap N=\mathfrak{m}^{n-s}\left(\mathfrak{m}^{s} M \cap N\right) ?
$$

In the same paper they already gave a negative answer to this problem (in the non-affine case) by building a 2-dimensional regular ring $A$ whose maximal ideals form a countable set, say $\left\{\mathfrak{m}_{1}, \mathfrak{m}_{2}, \ldots\right\}$, such that $\cap_{i} \mathfrak{m}_{i}$ is a nonzero principal prime ideal generated by an element $f$, where $f \in \mathfrak{m}_{i}^{i}$ for every $i \geq 1$. In particular and since $A$ is a domain, $\mathfrak{m}_{i}\left(\mathfrak{m}_{i}^{i-1} \cap(f)\right)=$ $\mathfrak{m}_{i}(f) \subsetneq(f)$ whereas $\mathfrak{m}_{i}^{i} \cap(f)=(f)$.

An answer to a slightly weaker version of this question was given by O'Carroll in [19] under the hypothesis of $A$ being an excellent ring.

TheOrem 2.2 [19] Let $A$ be an excellent ring and let $N \subseteq M$ be two finitely generated $A$-modules. Then there exists an integer $s \geq 1$ such that, for all integers $n \geq s$ and for all maximal ideals $\mathfrak{m}$ of $A$,

$$
\mathfrak{m}^{n} M \cap N \subset \mathfrak{m}^{n-s} N .
$$

And the full answer to the question of Eisenbud and Hochster was given two years later by Duncan and O'Carroll. 
Theorem 2.3 [6] Let $A$ be an excellent ring and let $N \subseteq M$ be two finitely generated $A$-modules. Then there exists an integer $s \geq 1$ such that, for all integers $n \geq s$ and for all maximal ideals $\mathfrak{m}$ of $A$,

$$
\mathfrak{m}^{n} M \cap N=\mathfrak{m}^{n-s}\left(\mathfrak{m}^{s} M \cap N\right) .
$$

Later on, O'Carroll showed the uniformity of the Artin-Rees property for the set of principal ideals of a noetherian ring.

THEOREM 2.4 [20] Let $A$ be a noetherian ring and let $N \subseteq M$ be two finitely generated $A$-modules. Then there exists an integer $s \geq 1$ such that, for all integers $n \geq s$ and for all elements $x$ of $A$,

$$
x^{n} M \cap N=x^{n-s}\left(x^{s} M \cap N\right) .
$$

And soon after, he noted that Theorem 2.4 allows for a quick proof of Theorem 2.2 in the case of an affine ground ring over a perfect field (see $[21])$.

\section{Uniform Artin-Rees on arbitrary sets of ideals}

The results of O'Carroll ([19],[20]) and Duncan and O'Carroll ([6]) gave a complete answer to the question posed by Eisenbud and Hochster and, in the meantime, suggested new and interesting problems undertaken by Huneke in [11]. In order to facilitate the reading of the next results, we introduce a notation very close to the one proposed by Huneke in [11]. Given a noetherian ring $A$ and two finitely generated $A$-modules $N \subseteq M$, it is said that the pair $(N, M)$ has the strong (weak) uniform Artin-Rees property with respect to a set of ideals $\mathcal{W}$ of $A$ and with strong (weak) uniform Artin-Rees number $s$, the integer $s$ depending on $N, M$ and $\mathcal{W}$, if, for all integer $n \geq s$ and for every ideal $I$ of $\mathcal{W}, I^{n} M \cap N=I^{n-s}\left(I^{s} M \cap N\right)\left(I^{n} M \cap N \subseteq I^{n-s} N\right)$. Clearly, if $s$ is a uniform Artin-Rees number and $t$ is an integer such that $t \geq s$, then $t$ is also a uniform Artin-Rees number. The minimum of the strong (weak) uniform Artin-Rees numbers is denoted by $s=s(N, M ; \mathcal{W})$ $(w=w(N, M ; \mathcal{W}))$. If every pair $N \subset M$ of finitely generated $A$-modules has the strong (weak) uniform Artin-Rees property with respect to $\mathcal{W}$ it is said that $A$ has the strong (weak) uniform Artin-Rees property with respect to $\mathcal{W}$. If $\mathcal{W}$ is the set of all ideals of $A$, then the phrase "with respect to $\mathcal{W}$ " is deleted in these definitions and one writes $s=s(N, M)(w=w(N, M))$.

In [11] (Theorems 4.12 and 5.11), and with the aid of the tight closure theory, Huneke strengthened the study of the uniform Artin-Rees properties to the whole set of ideals of $A$ by weakening the thesis from the strong uniform Artin-Rees property to the weak uniform Artin-Rees property. 
THEOREM 3.1 [11] Let $A$ be a noetherian ring and let $N \subset M$ be two finitely generated A-modules. If A satisfies at least one of the conditions below, then there exists an integer $s \geq 1$ such that, for all integers $n \geq s$ and for all ideals $I$ of $A$,

$$
I^{n} M \cap N \subseteq I^{n-s} N
$$

(i) A is essentially of finite type over a noetherian local ring.

(ii) $A$ is of characteristic $p$ and $A$ is a finite module over $A^{p}$.

(iii) A is essentially of finite type over $\mathbb{Z}$.

(iv) $A$ is an excellent 3-dimensional domain.

And in the same paper he conjectured that excellent noetherian rings of finite Krull dimension have the weak uniform Artin-Rees property (see [11], Conjecture 1.3). Recently, Huneke showed a new case in which the conjecture holds.

THEOREM 3.2 [13] Let $A$ be an excellent noetherian ring which is the homomorphic image of a regular ring of finite Krull dimension. Assume that for all primes $\mathfrak{p}$ of $A$, the integral closure of $A / \mathfrak{p}$ has a resolution of singularities obtained by blowing up an ideal. Then $A$ has the weak uniform Artin-Rees property.

\section{The Relation Type Conjecture}

But the search for uniform properties in noetherian rings has been far from being circumscribed to the Artin-Rees properties. Uniform bounds in the theory of test elements ([12]), uniform Briançon-Skoda theorems ([11],[12]), uniform bounds for annihilators of local cohomology ([27]) or uniform bounds for the relation type of parameters ideals ([12], [15], [42]) are some examples of the great interest awakened by the subject. It is the last one which we we want to survey now.

Before that, let us revise the definition of relation type of an ideal (see, for instance, [14], [15], [24], [28], [32], [35], for definitions and properties). Let $A$ be noetherian ring and let $I$ be an ideal of $A$ generated by elements $x_{1}, \ldots, x_{n}$ of $A$. Let $\varphi: A\left[T_{1}, \ldots, T_{n}\right] \rightarrow \mathcal{R}(I)=A[I t]$ be the natural homogenous map which sends $T_{i}$ to $x_{i} t$. The relation type of $I, \operatorname{rt}(I)$, is the largest degree of any minimal system of generators of the kernel of $\varphi$. The ideal $I$ is said to be an ideal of linear type if $\operatorname{rt}(I)=1$ (see [8] and the references there). An already classical result concerning ideals of linear type, proved simultaneously by Huneke [9] and Valla [38] (see [34] for the case of modules), 
is that ideals generated by $d$-sequences are of linear type. Regular sequences are examples of $d$-sequences. It is well-known that in a Cohen-Macaulay local ring any system of parameters forms a regular sequence, in particular, a $d$ sequence. In fact, Buchsbaum local rings are the rings in which every system of parameters is a $d$-sequence $([10])$. Therefore, in Buchsbaum rings every ideal generated by a system of parameters has relation type 1 . Moreover, the relation type is controlled through faithfully flat extensions and the quotient by modules of finite length (see, e.g., [24], [25] and [42]). From this point of view it is natural to ask the following question:

Question 4.1 Relation-Type Conjecture [12]. Let $A$ be a complete local equidimensional noetherian ring. Does there exist an integer $s \geq 1$ such that, for every ideal I of A generated by a system of parameters, the relation type of $I$ is bounded above by $s$ ?

Wang in [41] first proved this conjecture for 2-dimensional noetherian local rings. Concretely:

TheOrem 4.2 [41] Let $A$ be a 2-dimensional noetherian local ring. Then there exists an integer $s \geq 1$ such that, for every ideal I of A generated by a system of parameters, the relation type of $I$ is bounded above by $s$.

And afterwards, he proved the conjecture for rings with finite local cohomology (see [42]).

THEOREM 4.3 [42] Let $(A, \mathfrak{m})$ be a d-dimensional noetherian local ring such that $H_{\mathfrak{m}}^{i}(A)$ is finitely generated for $i=0, \ldots, d-1$. Then there exist an integer $s \geq 1$ such that, for every ideal $I$ of $A$ generated by a system of parameters, the relation type of $I$ is bounded above by $s$.

For rings of dimension 3 or more, see the very recent work of Aberbach, Ghezzi and Ha [1].

\section{A long-time known connection}

The clear connection between the Artin-Rees lemma and the notion of relation type, at least for the case of ideals of linear type, appeared explicitly in the following two results. The first one is due to Huneke ([9]) and states that an ideal $I$ such that its extension $I+J / J$ on the quotient ring $A / J$ is generated by a $d$-sequence verifies a certain Artin-Rees property.

Proposition 5.1 [9] Let $A$ be a commutative ring. If $J$ is an ideal of $A$ and $I$ is an ideal of $A$ generated by a d-sequence modulo $J$, then for all integers $n \geq 1$,

$$
I^{n} \cap J \subset I^{n-1} J
$$


The second one is due to Herzog, Simis and Vasconcelos ([8]) and is what they called the "Artin-Rees lemma on the nose". There, the hypothesis was on both ideals $I$ and $I / J$.

Proposition 5.2 [8] Let $A$ be a commutative ring. If $J \subset I$ are two ideals of $A$ such that $I$ and $I / J$ are ideals of linear type, then for all integers $n \geq 1$,

$$
I^{n} \cap J=I^{n-1} J
$$

Much later, and already in the context of uniform properties, Lai proved in [15] that the Relation Type Conjecture for the quotient ring of a Buchsbaum local ring $A$ by an ideal $J$ is equivalent to the strong uniform ArtinRees property for the pair $(J, A)$ and with respect to the set of ideals of $A$ whose image in $A / J$ is a parameter ideal. Concretely:

TheOrem 5.3 [15] Let $A$ be a Buchsbaum local ring and let $J$ be an ideal of $A$. Then the following are equivalent:

(i) The pair $(J, A)$ has the strong uniform Artin-Rees property with respect to the set of ideals of $A$ whose image in $A / J$ is a parameter ideal of $A / J$ and with strong uniform Artin-Rees number s.

(ii) The relation type of parameter ideals of $A / J$ is uniformly bounded by $s$.

A similar result to this was proved by Wang in [41] where a bound for the relation type of an ideal is deduced from a strong uniform Artin-Rees number. Concretely,

Proposition 5.4 [41] Let $A$ be a noetherian local ring and let I be an ideal of $A$ generated by a regular sequence. If $J$ is an ideal of $A$ such that, for all integers $n \geq s, I^{n} \cap J=I^{n-s}\left(I^{s} \cap J\right)$, then $\operatorname{rt}(I+J / J) \leq s$.

And using this fact, he was able to prove that the strong uniform ArtinRees property cannot hold for the class of all ideals, even in a 3-dimensional regular local ring, by displaying a family of ideals with unbounded relation type in a 2-dimensional regular local ring ([41]).

EXAmPLE 5.5 [41] Let $k$ be a field and let $A=k \llbracket X, Y, Z \rrbracket$ be the power series ring in three variables. Let $J=(Z)$ be the ideal of $A$ generated by $Z$. For each integer $r \geq 1$, consider the ideal $I_{r}=\left(X^{r}, Y^{r}, X^{r-1} Y+Z^{r}\right)$ of $A$, which is generated by a regular sequence. Clearly $\operatorname{rt}\left(I_{r}+J / J\right) \geq r$. In particular, there does not exist an integer $s \geq 1$ such that, for all integers $n \geq s$ and for all integers $r \geq 1$,

$$
I_{r}^{n} \cap J=I_{r}^{n-s}\left(I_{r}^{s} \cap J\right) .
$$




\section{The exact sequence linking AR with RT}

So far we have seen that the strong uniform Artin-Rees property holds for the set of maximal ideals and the set of principal ideals; that the weak ArtinRees property holds for the whole set of ideals under some conditions on the base ring; and that Artin-Rees theory and Relation Type are two linked subjects so that questions on uniform Artin-Rees may be translated into questions on relation type and vice versa, for instance, the Relation Type Conjecture or that the strong uniform Artin-Rees property does not hold for the whole class of ideals of a ring. Our next purpose now is to deepen on this link. In order to do that, we recall some notations and properties beginning with the (module of effective) relations of the Rees module of an ideal $I$ with respect to a module $M$ (see [25]).

If $A$ is a commutative ring, we mean by a standard $A$-algebra a commutative graded algebra $U=\oplus_{n \geq 0} U_{n}$, with $U_{0}=A$ and $U$ generated by the elements of degree 1 . Two examples of standard algebras are the Rees algebra $\mathcal{R}(I)$ of an ideal $I$ of $A$, which is a standard $A$-algebra, and the associated graded ring $\mathcal{G}(I)$ of $I$, which is a standard $A / I$-algebra. Let $U_{+}=\oplus_{n>0} U_{n}$ be the irrelevant ideal of $U$. If $E=\oplus_{n \geq 0} E_{n}$ is a graded $U$-module, let $s(E)=\min \left\{r \geq 1 \mid E_{n}=0\right.$ for all $\left.n \geq r+1\right\}$, which may possibly be infinite.

A standard $U$-module will be a graded $U$-module $F=\oplus_{n \geq 0} F_{n}$ such that $F_{n}=U_{n} F_{0}$ for all $n \geq 0$. The Rees module $\mathcal{R}(I ; M)=\oplus_{n \geq 0} I^{n} M$ of an ideal $I$ of $A$ with respect to an $A$-module $M$ is a standard $\mathcal{R}(I)$-module and the associated graded module $\mathcal{G}(I ; M)=\oplus_{n \geq 0} I^{n} M / I^{n+1} M$ of $I$ with respect to an $A$-module $M$ is a standard $\mathcal{G}(I)$-module.

Given $F$ and $G$, two standard $U$-modules, and $\varphi: G \rightarrow F$, a surjective graded morphism of $U$-modules, $E(\varphi)$ denotes the graded $A$-module $E(\varphi)=$ $\operatorname{ker} \varphi / U_{+} \operatorname{ker} \varphi=\operatorname{ker} \varphi_{0} \oplus\left(\oplus_{n \geq 1} \operatorname{ker} \varphi_{n} / U_{1} \operatorname{ker} \varphi_{n-1}\right)=\oplus_{n \geq 0} E(\varphi)_{n}$. The next remark is a simple but very useful property, which will be decisive in what follows: if $\varphi: G \rightarrow F, \psi: H \rightarrow G$ are two surjective graded morphisms of graded $U$-modules, then there exists a graded exact sequence of $A$-modules

$$
E(\psi) \rightarrow E(\varphi \circ \psi) \stackrel{\psi}{\rightarrow} E(\varphi) \rightarrow 0 .
$$

In particular, $s(E(\varphi)) \leq s(E(\varphi \circ \psi)) \leq \max (s(E(\varphi)), s(E(\psi))$ ) (see Lemma 2.3 in [25]).

For a standard $U$-module $F$ and $\gamma: \mathbf{S}\left(U_{1}\right) \otimes F_{0} \stackrel{\alpha \otimes 1}{\longrightarrow} U \otimes F_{0} \rightarrow F$, where $\alpha: \mathbf{S}\left(U_{1}\right) \rightarrow U$ is the canonical symmetric presentation of $U$ and $U \otimes F_{0} \rightarrow F$ is the structural morphism, the module of effective $n$-relations of $F$ is defined to be $E(F)_{n}=E(\gamma)_{n}=\operatorname{ker} \gamma_{n} / U_{1} \operatorname{ker} \gamma_{n-1}$ (where, for $n=0, E(F)_{n}=0$ ). Put $E(F)=\oplus_{n \geq 1} E(F)_{n}=\oplus_{n \geq 1} E(\gamma)_{n}=E(\gamma)=\operatorname{ker} \gamma / \mathbf{S}_{+}\left(U_{1}\right) \operatorname{ker} \gamma$. The relation type of $F$ is defined to be $\operatorname{rt}(F)=s(E(F))$, that is, $\operatorname{rt}(F)$ is the 
minimum integer $r \geq 1$ such that the effective $n$-relations are zero for all $n \geq r+1$. Using the exact sequence (1), it can be shown that the module of effective $n$-relations and the relation type do not depend on the chosen symmetric presentation ([25]).

For an ideal $I$ of $A$ and an $A$-module $M$, the module of effective $n$ relations and the relation type of $I$ with respect to $M$ are defined to be $E(I ; M)_{n}=E(\mathcal{R}(I ; M))_{n}$ and $\operatorname{rt}(I ; M)=\operatorname{rt}(\mathcal{R}(I ; M))$ (see also [8], page 106, and [34], page 41). Analogously to what happens with the Castelnuovo regularity ([22]), with the symmetric canonical presentations ([38]) and with further work on $a$-invariants ([36]), the relation type of $I$ with respect to $M$ coincides with the relation type of $\mathcal{G}(I ; M)([25])$.

Now, given an ideal $I$ of $A$ and two $A$-modules $N \subset M$, take the standard $\mathbf{S}(I)$-modules $F=\mathcal{R}(I ; M / N), G=\mathcal{R}(I ; M)$ and $H=\mathbf{S}(I) \otimes M$. Consider the surjective graded morphisms of $\mathbf{S}(I)$-modules $\varphi: G \rightarrow F$ defined by $\varphi_{n}$ : $G_{n}=I^{n} M \rightarrow I^{n} M / I^{n} M \cap N=I^{n} M+N / N=F_{n}$ and $\psi: H \rightarrow G$ induced by the natural graded morphism $\alpha: \mathbf{S}(I) \rightarrow \mathcal{R}(I)$. Since $\psi$ is a symmetric presentation of $G=\mathcal{R}(I ; M), E(\psi)=E(I ; M)$ and $s(E(\psi))=\operatorname{rt}(I ; M)$. Since $\varphi \circ \psi$ is a symmetric presentation of $F=\mathcal{R}(I ; M / N), E(\varphi \circ \psi)=$ $E(I ; M / N)$ and $s(E(\varphi \circ \psi))=\operatorname{rt}(I ; M / N)$. Finally, it is clear that $E(\varphi)_{n}=$ $I^{n} M \cap N / I\left(I^{n-1} M \cap N\right)$, which coincides with the module $E(N, M ;\{I\})_{n}$ introduced in Section 1. In particular, $s(E(\varphi))=s(N, M ;\{I\})$. Using the exact sequence (1), we arrive at the desired exact sequence of $A$-modules which links Artin-Rees theory with relation type (see [25]):

$$
E(I ; M)_{n} \rightarrow E(I ; M / N)_{n} \rightarrow E(N, M ;\{I\})_{n} \rightarrow 0 .
$$

Evaluating the "function" $s$ on (2), we obtain the inequalities:

$$
s(N, M ;\{I\}) \leq \operatorname{rt}(I ; M / N) \leq \max (\operatorname{rt}(I ; M), s(N, M ;\{I\})) .
$$

\section{Uniform Relation Type}

The purpose of this section is to prove the results of Section 5 using the inequalities (3). Let us begin with the results of Huneke and of Herzog, Simis and Vasconcelos.

Proof of Proposition 5.1 and Proposition 5.2: If $I$ is of linear type modulo $J$ (for instance, if it is generated by a $d$-sequence modulo $J$ ), then $\operatorname{rt}(I+J / J)=1$. From the first inequality in $(3), s(J, A ;\{I\}) \leq \operatorname{rt}(I ; A / J)=$ 1. In other words $I^{n} \cap J=I^{n-1}(I \cap J)$ for all $n \geq 1$. In particular, $I^{n} \cap J \subset I^{n-1} J$ and, if $J \subset I, I^{n} \cap J=I^{n-1} J$. 
Remark that the hypothesis $I$ being generated by a $d$-sequence modulo $J$ has been weakened to $I$ being of linear type modulo $J$ and that the hypothesis $I$ being of linear type is not needed. With respect to the result of Wang, we have the following:

Proof of Proposition 5.4: If $I$ is of linear type (for instance, if $I$ is generated by a regular sequence), $\operatorname{rt}(I ; A)=1$. If $I^{n} \cap J=I^{n-s}\left(I^{s} \cap J\right)$ for all $n \geq s$, then $s(J, A ;\{I\}) \leq s$. By the second inequality in (3), we obtain that $\operatorname{rt}(I ; A / J) \leq \max (\operatorname{rt}(I ; A), s(J, A ;\{I\})) \leq s$. So $\operatorname{rt}(I+J / J) \leq s$.

Before giving a proof of the result of Lai, let us introduce the following useful notation which is in some sense implicit in everything we have been doing before.

Given a commutative ring $A$ and an $A$-module $M$, the uniform relation type of $M$ with respect to a set of ideals $\mathcal{W}$ of $A$ is defined to be $\operatorname{urt}(M ; \mathcal{W})=$ $\sup \{\operatorname{rt}(I ; M) \mid I \in \mathcal{W}\}$. If $\mathcal{W}$ is the set of all ideals of $A$, then the phrase "with respect to $\mathcal{W}$ " is deleted and we simply write urt $(M)$. Taking suprema in the key inequalities (3), one has

$$
s(N, M ; \mathcal{W}) \leq \operatorname{urt}(M / N ; \mathcal{W}) \leq \max (\operatorname{urt}(M ; \mathcal{W}), s(N, M ; \mathcal{W})) .
$$

In other words, one way to prove a strong uniform Artin-Rees property for a pair $(N, M)$ with respect to a set of ideals $\mathcal{W}$ consists in bounding above the uniform relation type of the quotient $M / N$ with respect to the same set of ideals.

Now, we prove the result of Lai:

Proof of Theorem 5.3: Let $\mathcal{V}$ be the set of parameters ideals $I$ of $A$ such that $I+J / J$ is a parameter ideal of $A / J$ and let $\mathcal{W}$ be the set of ideals $I$ of $A$ such that $I+J / J$ is a parameter ideal of $A / J$. Clearly $\mathcal{V} \subset \mathcal{W}$ and $s(J, A ; \mathcal{V}) \leq s(J, A ; \mathcal{W})$. If $I \in \mathcal{W}$, then, by prime avoidance, one can find an ideal $K$ of $A$ such that $K$ is generated by a system of parameters of $A$ and $K+$ $J / J=I+J / J$. In particular $\operatorname{rt}(I+J / J)=\operatorname{rt}(K+J / J)$ and $\operatorname{urt}(A / J ; \mathcal{W})=$ $\operatorname{urt}(A / J ; \mathcal{V})$. On the other hand, since $A$ is a Buchsbaum local ring, wherein parameters ideals are generated by $d$-sequences, $\operatorname{urt}(A ; \mathcal{V})=1$. Using the inequalities (4), we obtain:

$$
\begin{aligned}
& s(J, A ; \mathcal{V}) \leq s(J, A ; \mathcal{W}) \leq \operatorname{urt}(A / J ; \mathcal{W})=\operatorname{urt}(A / J ; \mathcal{V}) \text { and } \\
& \operatorname{urt}(A / J ; \mathcal{V}) \leq \max (\operatorname{urt}(A ; \mathcal{V}), s(J, A ; \mathcal{V}))=s(J, A ; \mathcal{V})
\end{aligned}
$$

Thus $s(J, A ; \mathcal{W})=\operatorname{urt}(A / J ; \mathcal{W})$, proving the equivalence between the strong uniform Artin-Rees property for the pair $(J, A)$ with respect to the set of parameter ideals modulo $J$ and the relation type conjecture for the ring $A / J$.

From now on we will focus our attention on bounding the uniform relation type. 


\section{Uniform Relation Type in dimension 1}

Since the strong uniform Artin-Rees property cannot hold for the class of all ideals, there will not be in general a uniform bound for the relation type of all ideals of a ring. So the first question is to characterize which are the rings $A$ where $\operatorname{urt}(A)$ is finite. A particular case was solved by Costa in [5] where he proved that for a commutative (non necessarily noetherian local) domain $A, \operatorname{urt}(A)=1$ is equivalent to $A$ being a Prüfer domain. And a little more generally, commutative rings $A$ with $\operatorname{urt}(A)=1$ are known to be the rings of weak dimension one or less ([23]). In particular, for a notherian local $\operatorname{ring} A, \operatorname{urt}(A)=1$ if and only if $A$ is a discrete valuation ring or a field. But if the intention is to characterize just the finiteness of urt $(A)$, then the works of Cohen [4] and Sally [30], wherein noetherian local rings with a uniform bound on the number of generators of all ideals are characterized as the rings of Krull dimension at most one, might be a source of inspiration. And indeed, the following result goes in the same direction.

THEOREM 8.1 [25] Let $A$ be an excellent ring. The following conditions are equivalent:

(i) $\operatorname{urt}(M)<\infty$ for all finitely generated $A$-modules $M$.

(ii) $\operatorname{urt}(A)<\infty$.

(iii) There exists an integer $s \geq 1$ such that, for every three-generated ideal $I$ of $A$, the relation type of $I$ is bounded by $s$.

(iv) $\operatorname{dim} A \leq 1$.

In particular, and using the inequalities (4), we have the strong uniform Artin-Rees property in codimension one.

Corollary 8.2 [25] Let $A$ be an excellent ring and let $N \subseteq M$ be two finitely generated $A$-modules such that $\operatorname{dim}(M / N) \leq 1$. Then there exists an integer $s \geq 1$ such that, for all integers $n \geq s$ and for all ideals $I$ of $A$,

$$
I^{n} M \cap N=I^{n-s}\left(I^{s} M \cap N\right) .
$$

With respect to the proof of Theorem 8.1, it is clear that $(i) \Rightarrow(i i) \Rightarrow$ (iii). The proof of $(i i i) \Rightarrow(i v)$ takes into account that in a 2-dimensional (or greater) noetherian local ring $(A, \mathfrak{m})$, there exist two $\mathfrak{m}$-independent elements $x, y \in A$ (see, for instance, [37]). With these two elements, one can consider the family of ideals of $A$ defined by $I_{r}=\left(x^{r}, y^{r}, x^{r-1} y\right)$ (remark that, modulo $J$, it is the same family considered by Wang in Example 5.5). Then, it can easily be checked that the module of effective $r$-relations of $I_{r}$ is generated 
by the class of the polynomial $T_{1}^{r-1} T_{2}-T_{3}^{r}$ and that the relation type of $I_{r}$ is exactly $r$. The proof of $(i v) \Rightarrow(i)$ consists in reducing the problem to a 1-dimensional maximal Cohen-Macaulay module. In this case, if $I$ is an $\mathfrak{m}$-primary ideal, then the relation type of $I$ with respect to $M$ is bounded above by $e(A)$, the multiplicity of $A$ (see [25] for the details). For instance, if $A$ is a 1-dimensional noetherian local reduced ring, one proves that $\operatorname{urt}(A) \leq e(A)+1$. And if $A$ is a domain, then $\operatorname{urt}(A) \leq e(A)$ (see, for instance, [25] and [31] for more examples).

\section{Uniform Relation Type with respect to maximal ideals}

Having in mind the strong uniform Artin-Rees property with respect to some sets of ideals, it was shown in [26] that the uniform relation type with respect to the set of all maximal ideals of an excellent ring is finite (see also [33] for another proof).

THEOREM 9.1 [33], [26] Let $A$ be an excellent ring and let $M$ be a finitely generated A-module. Then there exists an integer $s \geq 1$ such that, for all maximal ideals $\mathfrak{m}$ of $A$, the relation type of $\mathfrak{m}$ with respect to $M$ satisfies

$$
\operatorname{rt}(\mathfrak{m} ; M) \leq s .
$$

In particular, and using again the inequalities (4), one can recover Theorem 2.3 due to Duncan and O'Carroll.

Corollary 9.2 [6] Let $A$ be an excellent ring and let $N \subseteq M$ be two finitely generated $A$-modules. Then there exists an integer $s \geq 1$ such that, for all integers $n \geq s$ and for all maximal ideals $\mathfrak{m}$ of $A$,

$$
\mathfrak{m}^{n} M \cap N=\mathfrak{m}^{n-s}\left(\mathfrak{m}^{s} M \cap N\right) .
$$

The proof of Theorem 9.1 has three main steps. The first is a characterization of when the natural surjective morphism between the tensor product of two associated graded rings with the associated graded ring of the sum is an isomorphism, in terms of normal transversality (see also [39], pages 130 and following). Concretely, if $I$ and $J$ are two ideals of a noetherian ring $A$, it is shown that $\mathcal{G}(I) \otimes \mathcal{G}(J) \simeq \mathcal{G}(I+J)$ if and only if $\operatorname{Tor}_{i}\left(A / I^{p}, A / J^{q}\right)=0$, for all integers $p, q \geq 1$ and for the integers $i=1,2$. In the second step, one proves that the relation type of the tensor product of two standard modules is bounded above by the maximun of the relation type of each one, that is, $\operatorname{rt}(F \otimes G) \leq \max (\operatorname{rt}(F), \operatorname{rt}(G))$, being $F$ and $G$ two standard modules. Then the third step is obtained from the first two. In fact one deduces that if 
$(A, \mathfrak{m})$ is a noetherian local ring, if $M$ is a finitely generated $A$-module and if $\mathfrak{p}$ is a prime ideal such that $A / \mathfrak{p}$ is regular local and $\mathcal{G}(\mathfrak{p})$ and $\mathcal{G}(\mathfrak{p} ; M)$ are free $A / \mathfrak{p}$-modules, then

$$
\operatorname{rt}(\mathfrak{m}, M) \leq \operatorname{rt}(\mathfrak{p} ; M) .
$$

To finish the whole proof, one constructs, by using an argument involving generic flatness (see Theorem 22.A [16]), a cover of the spectrum of the ring $A$ by finitely many locally closed sets where the hypotheses of the third step are fulfilled.

\section{Uniform Relation Type with respect to princi- pal ideals}

Another set of ideals in which there is a known bound for the uniform relation type is the set of principal ideals of a noetherian ring.

THEOREM 10.1 [25] Let $A$ be an excellent ring and let $M$ be a finitely generated $A$-module. Then there exists an integer $s \geq 1$ such that, for all element $x \in A$, the relation type of $(x)$ with respect to $M$ satisfies

$$
\operatorname{rt}((x) ; M) \leq s .
$$

And from inequalities (4), one can deduce Theorem 2.4 due to O'Carroll.

Corollary 10.2 [20] Let $A$ be a noetherian ring and let $N \subseteq M$ be two finitely generated $A$-modules. Then there exists an integer $s \geq 1$ such that, for all integers $n \geq s$ and for all elements $x$ of $A$,

$$
x^{n} M \cap N=x^{n-s}\left(x^{s} M \cap N\right) .
$$

The proof of Theorem 10.1 is essentially the same as the one given by O'Carroll in his work ([20]). It consists in taking a minimal primary decomposition of 0 in $M, Q_{1} \cap \ldots \cap Q_{r}=0$, and then realizing that $\mathrm{rt}((x) ; M) \leq s$, for an integer $s \geq 1$ such that every $Q_{i}$ contains $r_{M}\left(Q_{i}\right)^{s} M$.

\section{Acknowledgments}

I would like to thank the organizers of the Lisbon Conference on Commutative Algebra for giving me the opportunity to participate and enjoy the conference. Special thanks are due to Ana Luisa Correia, Maria Vaz Pinto and Santiago Zarzuela for their willingness to help and provide a very comfortable atmosphere for work in this wonderful and friendly city. To all of them: muito obrigado. I am also grateful to Philippe Gimenez, Cristodor 
Ionescu, José M. Giral and Wolmer V. Vasconcelos for their helpful comments on the subject of this talk. Finally, I would also like to thank the referee for the careful reading of this paper. This work has been partially supported by the BFM2001-3584 grant.

\section{References}

[1] I.M. Aberbach, L. Ghezzi, H.T. Ha, Bounding the relation type of parameters ideals, Preprint.

[2] M.F. Atiyah, I.G. Macdonald, Introduction to Commutative Algebra, Addison-Wesley Publishing Co., Reading, 1969.

[3] N. Bourbaki, Commutative Algebra. Chapters 1-7. Translated from the French. Elements of Mathematics. Springer-Verlag, Berlin, 1989.

[4] I.S. Cohen, Commutative rings with restricted minimum condition, Duke Math. J. 17 (1950), 27-42.

[5] D. Costa, On the torsion-freeness of the symmetric powers of an ideal, J. Algebra 80 (1983), 152-158.

[6] A.J. Duncan, L. O'Carroll, A full uniform Artin-Rees theorem, J. reine angew. Math. 394 (1989), 203-207.

[7] D. Eisenbud, M. Hochster, A Nullstellensatz with nilpotents and Zariski's main lemma on holomorphic functions, J. Algebra 58 (1979), 157-161.

[8] J. Herzog, A. Simis, W.V. Vasconcelos, Koszul homology and blowingup rings, Lecture Notes in Pure and Appl. Math. 84 (1982), pp. 79-169. Marcel Dekker, New York.

[9] C. Huneke, On the symmetric and Rees algebra of an ideal generated by a $d$-sequence, J. Algebra 62 (1980), 268-275.

[10] C. Huneke, The theory of $d$-sequences and powers of ideals, Adv. in Math. 46 (1982), no. 3, 249-279.

[11] C. Huneke, Uniform bounds in noetherian rings, Invent. Math. 107 (1992), 203-223.

[12] C. Huneke, Tight closure and its applications, CBMS Lecture Notes 88 (1996), American Mathematical Society, Providence.

[13] C. Huneke, Desingularizations and the uniform Artin-Rees theorem, J. London Math. Soc. (2) 62 (2000), 740-756. 
[14] B. Johnson, D. Katz, On the relation type of large powers of an ideal, Mathematika 41 (1994), 209-214.

[15] Y.H. Lai, On the relation type of systems of parameters, J. Algebra 175 (1995), 339-358.

[16] H. Matsumura, Commutative Algebra, Second Edition, Mathematics Lecture Note Series, Reading, Massachusetts 1980.

[17] H. Matsumura, Commutative Ring Theory, Cambridge University Press, 1986.

[18] M. Nagata, Local Rings, Interscience, 1962.

[19] L. O'Carroll, A uniform Artin-Rees theorem and Zariski's main lemma on holomorphic functions, Invent. Math. 90 (1987), 674-682.

[20] L. O'Carroll, A note on Artin-Rees numbers, Bull. London Math. Soc. 23 (1991), 209-212.

[21] L. O'Carroll, Addendum to: A note on Artin-Rees numbers, Bull. London Math. Soc. 23 (1991), 555-556.

[22] A. Ooishi, Genera and arithmetic genera of commutative rings, Hiroshima Math. J. 17 (1987), 47-66.

[23] F. Planas-Vilanova, Rings of weak dimension one and syzygetic ideals, Proc. Amer. Math. Soc. 124 (1996), 3015-3017.

[24] F. Planas-Vilanova, On the module of effective relations of a standard algebra, Math. Proc. Camb. Phil. Soc. 124 (1998), 215-229.

[25] F. Planas-Vilanova, The strong uniform Artin-Rees property in codimension one, J. reine angew. Math. 527 (2000), 185-201.

[26] F. Planas-Vilanova, Normal transversality and uniform bounds, J. London Math. Soc. (2) 62 (2000), 716-728.

[27] K.N. Raghavan, Uniform annihilation of local cohomology and of Koszul homology, Math. Proc. Camb. Phil. Soc. 112 (1992), 487-494.

[28] K.N Raghavan, Powers of ideals generated by quadratic sequences, Trans. Amer. Math. Soc. 343 (1994), 727-747.

[29] D. Rees, Two classical theorems of ideal theory, Proc. Camb. Phil. Soc. 52 (1956), 155-157.

[30] J.D. Sally, Number of generators of ideals in local rings, Lecture Notes in Pure and Appl. Math. 35 (1978), Marcel Dekker, New York. 
[31] J.D. Sally, W.V. Vasconcelos, Stable rings, J. Pure and Appl. Alg. 4 (1974), 319-336.

[32] P. Schenzel, Castelnuovo's index of regularity and reduction numbers, Banach Center Publications 26 (2) (1990), 201-208.

[33] V. Trivedi, Hilbert functions, Castelnuovo-Mumford regularity and uniform Artin-Rees numbers, Manuscripta Math. 94 (1997), 485-499.

[34] N.V. Trung, Absolutely superficial elements, Math. Proc. Camb. Phil. Soc. 93 (1983), 35-47.

[35] N.V. Trung, Reduction exponent and degree bound for the defining equations of graded rings, Proc. Amer. Math. Soc. 101 (1987), 229236 .

[36] N.V. Trung, The Castelnuovo regularity of the Rees algebra and the associated graded ring, Trans. Amer. Math. Soc. 350 (1998), 2813-2832.

[37] G. Valla, Elementi independenti rispetto ad un ideale, Rend. Sem. Mat. Univ. Padova 44 (1970), 339-354.

[38] G. Valla, On the symmetric and Rees algebras of an ideal, Manuscripta Math. 30 (1980), 239-255.

[39] W.V. Vasconcelos, Arithmetic of Blowup Algebras, Cambridge University Press, Cambridge, 1994.

[40] W.V. Vasconcelos, Computational Methods in Commutative Algebra and Algebraic Geometry, Springer-Verlag, Berlin, 1998.

[41] H.J. Wang, Some uniform properties of 2-dimensional local rings, J. Algebra 188 (1997), 1-15.

[42] H.J. Wang, The Relation-Type Conjecture holds for rings with finite local cohomology, Comm. in Algebra 25 (1997), 785-801.

[43] O. Zariski, A fundamental lemma from the theory of holomorphic functions on an algebraic variety, Ann. Mat. Pura Appl. (45) XXIX, 187-198 (1949). 